\title{
Low-energy Anti-neutrinos from the Sun
}

\author{
$\underline{\text { V.B. Semikoz }}^{*}$, S. Pastor ${ }^{\dagger}$ and J.W.F. Valle ${ }^{\ddagger}$ \\ ${ }^{a}$ Instituto de Física Corpuscular - C.S.I.C., Departament de Física Teòrica, Universitat de València \\ 46100 Burjassot, València, SPAIN
}

\begin{abstract}
If neutrino conversions within the Sun result in partial polarization of initial solar neutrino fluxes, then a new opportunity arises to observe the $\bar{\nu}_{e}$ 's in future neutrino experiments in the low energy region (such as BOREXINO or HELLAZ) and thus to probe the Majorana nature of the neutrinos. The $\nu_{e} \rightarrow \bar{\nu}_{e}$ conversions may take place for low energy solar neutrinos while being unobservable at the Kamiokande and Super-Kamiokande experiments.
\end{abstract}

Finding a signature for the Majorana nature of neutrinos or, equivalently, for the violation of lepton number in Nature is a fundamental challenge in particle physics [1]. All attempts for distinguishing Dirac from Majorana neutrinos directly in laboratory experiments have proven to be a hopeless task, due to the V-A character of the weak interaction, which implies that all such effects vanish as the neutrino mass goes to zero. We suggest an alternative way in which one might probe for the possibility of $L$-violation which is not directly induced by the presence of a Majorana mass. Although, Majorana masses will be required at some level, but the quantity which is directly involved is the transition amplitude for a $\nu_{e}$ to convert into an $\bar{\nu}_{e}$ inside the Sun.

Here we propose to probe for the possible existence of $L$-violating processes in the solar interior that can produce an $\bar{\nu}_{e}$ component in the neutrino flux. The idea is that, even though the nuclear reactions that occur in a normal star like our Sun do not produce directly right-handed active neutrinos $\left(\bar{\nu}_{a}\right)$ these may be produced by combining the chirality-flipping transition $\nu_{e L} \rightarrow \bar{\nu}_{a R}$

\footnotetext{
${ }^{*}$ On leave from IZMIRAN, Troitsk, Moscow region, 142092, Russia

${ }^{\dagger}$ E-mail: sergio@flamenco.ific.uv.es

${ }^{\ddagger}$ E-mail: $\quad$ valle@flamenco.ific.uv.es Work supported by DGICYT under grants PB95-1077 and SAB95506 (V.B.S.), by the TMR network grant ERBFMRXCT960090 and by INTAS grant 96-0659 of the European Union. S.P. was supported by Conselleria d'Educació i Ciència of Generalitat Valenciana. V.B.S. also acknowledges the support of RFFR through grants 97-02-16501 and 95-02-03724.
}

with the standard chirality-preserving MSW conversions $\nu_{e L} \rightarrow \nu_{\mu L}$ through cascade conversions like $\nu_{e L} \rightarrow \bar{\nu}_{\mu R} \rightarrow \bar{\nu}_{e R}$ or $\nu_{e L} \rightarrow \nu_{\mu L} \rightarrow \bar{\nu}_{e R}$. These conversions arise as a result of the interplay of two types of mixing [2: one of them, matter-induced flavour mixing, leads to MSW resonant conversions which preserve the lepton number $L$, whereas the other is generated by the resonant interaction of a Majorana neutrino transition magnetic moment with the solar magnetic field [3]. This violates the $L$ symmetry by two units $(\Delta L= \pm 2)$ and is an explicit signature of the Majorana nature of the neutrino 溲.

We consider neutrino-electron scattering in future underground solar neutrino experiments in the low-energy region, below the threshold for $\bar{\nu}_{e}+p \rightarrow n+e^{+}$, such as is the case for $p p$ or ${ }^{7} \mathrm{Be}$ neutrinos. These should be measured in future real-time experiments such as HELLAZ [5] or BOREXINO [6] which will have low energy thresholds (100 keV and $250 \mathrm{keV}$, respectively). BOREXINO is designed to take advantage of the characteristic shape of the electron recoil energy spectrum from the ${ }^{7} \mathrm{Be}$ neutrino line, while the HELLAZ experiment is intended to measure the fundamental neutrinos of the $p p$ chain.

The complete expression for the differential cross section of the weak process $\nu e \rightarrow \nu e$, as a function of the electron recoil energy $T$, in the massless neutrino limit, can be written as

$$
\begin{aligned}
& \frac{d \sigma}{d T}(\omega, T)=\frac{2 G_{F}^{2} m_{e}}{\pi}\left[P_{e} h\left(g_{e L}, g_{R}\right)\right. \\
& \left.+P_{\bar{e}} h\left(g_{R}, g_{e L}\right)+P_{a} h\left(g_{a L}, g_{R}\right)+P_{\bar{a}} h\left(g_{R}, g_{a L}\right)\right]
\end{aligned}
$$


where $h(a, b) \equiv a^{2}+b^{2}(1-T / \omega)^{2}-a b m_{e} T / \omega^{2}$ and $g_{e L}=\sin ^{2} \theta_{W}+0.5, g_{a L}=\sin ^{2} \theta_{W}-0.5(a=$ $\mu, \tau)$ and $g_{R}=\sin ^{2} \theta_{W}$ are the weak couplings of the Standard Model, and $\omega$ is the energy of the incoming neutrino. The parameter $P_{e}$ in the equation above is the survival probability of the initial left-handed electron neutrinos, while $P_{\bar{e}}, P_{a}$ and $P_{\bar{a}}$ are the appearance probabilities of the other species, that may arise in the Sun as a result of the processes $\nu_{e L} \rightarrow \bar{\nu}_{e R}, \nu_{e L} \rightarrow \nu_{a L}$ or $\nu_{e L} \rightarrow \bar{\nu}_{a R}$, respectively. These parameters obey the unitarity condition $P_{e}(\omega)+P_{\bar{e}}(\omega)+P_{a}(\omega)+P_{\bar{a}}(\omega)=1$. In general they are obtained from the complete $4 \times 4$ evolution Hamiltonian describing the evolution of the neutrino system [4. They depend on the neutrino energy $\omega$, on the solar magnetic field through the parameter $\mu_{\nu} B_{\perp}$ and on the neutrino mixing parameters $\Delta m^{2}, \sin ^{2} 2 \theta$.

In the $L$-violating processes one has in general all four contributions shown in eq. (11). In contrast, in the case where lepton number is conserved (like in MSW conversions), the solar neutrino flux will consist of neutrinos, so only the first and third terms in eq. (1) contribute. It follows that the differential cross section will be different in the case where $\nu_{e}$ 's from the Sun get converted to electron $\bar{\nu}_{e}$ 's. Is it possible to measure this difference in future neutrino experiments?

The relevant quantity to be measured in neutrino scattering experiments is the energy spectrum of events, namely

$\frac{d N_{\nu}}{d T}=N_{e} \sum_{i} \phi_{0 i} \int_{\omega_{\min }(T)}^{\omega_{\max }} d \omega \lambda_{i}(\omega)\left\langle\frac{d \sigma}{d T}(\omega, T)\right\rangle(2)$

where $d \sigma / d T$ is given in eq. (11) and $N_{e}$ is the number of electrons in the fiducial volume of the detector 7 . The sum in the above equation is done over the solar neutrino spectrum, where $i$ corresponds to the different reactions $i=p p$, ${ }^{7} \mathrm{Be}$, pep,${ }^{8} \mathrm{~B}$..., characterized by an integral flux $\phi_{0 i}$ and a differential spectrum $\lambda_{i}(\omega)$ (for neutrinos coming from two-body reactions, one has $\left.\lambda_{i}(\omega)=\delta\left(\omega-\omega_{i}\right)\right)$. The lower limit for the neutrino energy is $\omega_{\min }(T)=1 / 2\left(T+\sqrt{T^{2}+2 m_{e} T}\right)$, while the upper limit $\omega_{\max }$ corresponds to the

${ }^{4}$ For simplicity we have taken in eq. (2) the efficiency of the detector as unity for energies above the threshold. maximum neutrino energy. In order to take into account the finite resolution in the measured electron recoil energy, we perform a Gaussian average of the cross section, indicated by $\langle\ldots\rangle$ in eq. (2)). For further details, see ref. 77.

We have calculated the averaged energy spectrum of events for the two experiments in the simple case where the parameters $P_{i}$ do not depend on the neutrino energy. Here we present in figure 1 our results for BOREXINO. The upper line corresponds to the case where one has no neutrino conversions $\left(P_{e}=1\right)$. When electron anti-neutrinos are present in the solar flux the results are the lines labelled with $\bar{\nu}_{e}$, calculated for the indicated value of $P_{e}$ and the corresponding amount of $\bar{\nu}_{a}$. The cases of $\nu_{e} \rightarrow \nu_{\mu, \tau}$ and $\nu_{e} \rightarrow \bar{\nu}_{\mu, \tau}$ are the lower lines with labels $\nu_{a}$ and $\bar{\nu}_{a}$, respectively.

One can see from figure 1 that it is possible to distinguish the case with $\bar{\nu}_{e}$ considering the behaviour of the cross section for low energies. It is the slope of the measured spectrum the key for recognizing the presence of $\bar{\nu}_{e}$ 's in the solar neutrino flux, and correspondingly the presence of $L$-violating processes which can only exist if neutrinos are Majorana particles.

The shortcoming of the above discussion is that we have neglected the energy dependence of the physical parameters $P_{i}$. One must calculate the averaged $\nu-e$ cross section using analytical expressions for $P_{i}=P_{i}(\omega)$. However, since the ${ }^{7} \mathrm{Be}$ neutrinos are mono-energetic, whatever the mechanism that produces the deficit is, their survival probability will take on a constant value $P_{e}\left(\omega_{B e}\right)$. Therefore one can apply directly the results we have obtained for constant $P_{i}$ for the range of electron recoil energy where the contribution of ${ }^{7} \mathrm{Be}$ neutrinos dominates. In ref. [7] we give a discussion of the experimental uncertainties.

There are however stringent bounds on the presence of solar $\bar{\nu}_{e}$ 's in the high energy region $\left({ }^{8} \mathrm{~B}\right)$. These would interact within the detector through the process $\bar{\nu}_{e}+p \rightarrow n+e^{+}$. This process, which has an energy threshold of $E_{\nu}=$ $m_{n}-m_{p}+m_{e} \simeq 1.8 \mathrm{MeV}$, has not been found to occur in the Kamiokande experiment [8,9], nor in the very recent data from Super-Kamiokande [10]. Also the results from the liquid scintillation 

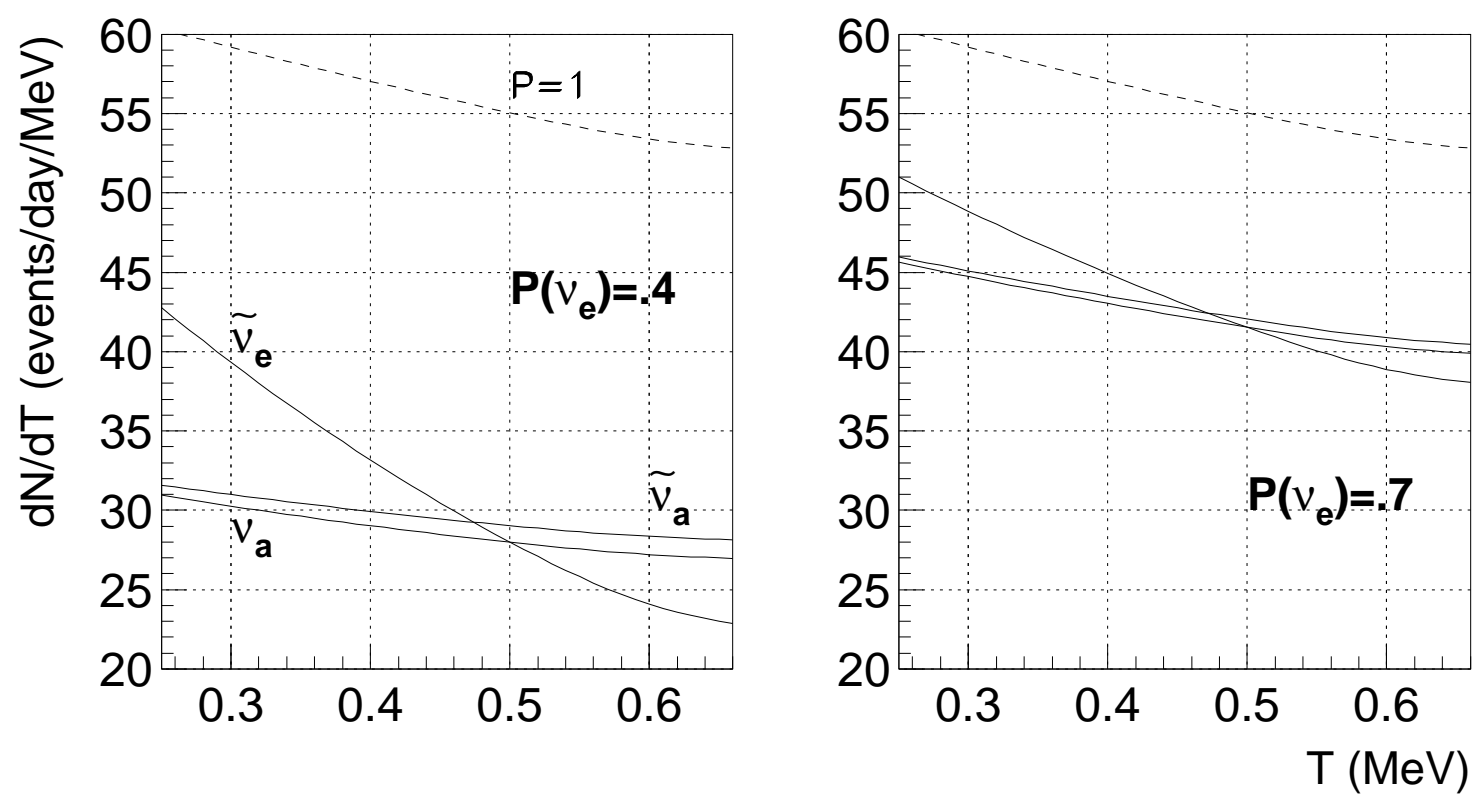

Figure 1. Energy spectrum of events corresponding to ${ }^{7}$ Be solar neutrinos for the BOREXINO experiment. Different cases are shown, as described in the text.

detector (LSD) are negative [11.

As we show in ref. [7] for the specific scenario presented in [2], the co-existence of a suppressed production of high-energy $\bar{\nu}_{e}$ 's and a sizeable flux of anti-neutrinos at energies below $1.8 \mathrm{MeV}$ can be easily understood theoretically. The resonance in the the $\nu_{e L} \rightarrow \bar{\nu}_{e R}$ conversions can lie in the energy region below $1 \mathrm{MeV}$ (relevant for HELLAZ or BOREXINO) provided that the neutrino parameters have reasonable values, so that the conversion probability is small for energies $\omega \gg 1$ $\mathrm{MeV}$. Therefore the anti-neutrino flux would be hidden in the background and therefore unobservable in Super-Kamiokande.

Our conclusion is that neutrino conversions within the Sun can result in partial polarization of the initial fluxes, in such a way as to produce a sizeable $\bar{\nu}_{e}$ component without conflicting present Super-Kamiokande data. The observation of $\bar{\nu}_{e}$ 's from the Sun in future neutrino experiments in the low energy region could lead to the conclusion that the neutrinos are Majorana particles.

\section{REFERENCES}

1. J.W.F. Valle, Prog. Part. Nucl. Phys. 26 (1991) 91, and references therein
2. E.Kh. Akhmedov, S.T. Petcov and A.Yu. Smirnov, Phys.Rev.D48 (1993) 2167; Phys. Lett. B309 (1993) 95

3. C.S. Lim and W.J. Marciano, Phys.Rev.D37 (1988) 1368; E.Kh. Akhmedov, Sov. Phys. JETP 68 (1989) 690

4. J. Schechter and J.W.F. Valle, Phys.Rev. D34 (1981) 1883; ibid. D25 (1982) 283

5. F. Arzarello et al., preprint CERN-LAA/9419; T. Ypsilantis et al, in Cosmological Dark Matter, (World Scientific, ISBN 98102-1879-6) 205; G. Bonvicini, Nucl. Phys. B (Proc.Suppl.) 35 (1994) 438

6. Status Report of BOREXINO Project: The Counting Test Facility and its Results. A proposal for participation in the BOREXINO Solar Neutrino Experiment, J.B. Benzinger et al., (Princeton, October 1996). Talk by P.A. Eisenstein at Baksan Int. School (April 1997)

7. S. Pastor, V.B. Semikoz and J.W.F. Valle, preprint hep-ph/9711316

8. R. Barbieri, G. Fiorentini, G. Mezzorani and M. Moretti, Phys. Lett. B259 (1991) 119

9. R. Raghavan et al, Phys.Rev.D44 (1991) 3786

10. G. Fiorentini, M. Moretti and F.L. Villante, preprint hep-ph/9707097

11. M. Aglietta et al., JETP Lett. 63 (1996) 791 